\title{
LAGO: the Latin American Giant Observatory
}

\author{
Iván Sidelnik ${ }^{1, *}$, Hernán Asorey ${ }^{2,3, * *}$, and for the LAGO Collaboration ${ }^{4}$ \\ ${ }^{1}$ Departamento de Física de Neutrones y Reactores, CONICET e Instituto Balseiro, Av. \\ Bustillo 9500, (8400) S.C. de Bariloche, Argentina. \\ ${ }^{2}$ Laboratorio Detección de Partículas y Radiación, CNEA e Instituto Balseiro, Av. Bustillo \\ 9500, (8400) S.C. de Bariloche, Río Negro, Argentina. \\ ${ }^{3}$ Instituto de Tecnologías en Detección y Astropartículas (CNEA, CONICET, UNSAM), Av. \\ Gral. Paz 1499, (1650) San Martín, Buenos Aires, Argentina \\ ${ }^{4}$ The Latin American Giant Observatory, lagoproject.org, see the full list of members and \\ institutions at lagoproject.org/collab.html \\ *Corresponding author, sidelnik@cnea.gov.ar \\ *Corresponding author, asoreyh@cab.cnea.gov.ar
}

March 17, 2017

\begin{abstract}
The Latin American Giant Observatory (LAGO) is an extended cosmic ray observatory composed of a network of water-Cherenkov detectors (WCD) spanning over different sites located at significantly different altitudes (from sea level up to more than $5000 \mathrm{~m}$ a.s.1.) and latitudes across Latin America, covering a wide range of geomagnetic rigidity cut-offs and atmospheric absorption/reaction levels. The LAGO WCD is simple and robust, and incorporates several integrated devices to allow time synchronization, autonomous operation, on board data analysis, as well as remote control and automated data transfer.

This detection network is designed to make detailed measurements of the temporal evolution of the radiation flux coming from outer space at ground level. LAGO is mainly oriented to perform basic research in three areas: high energy phenomena, space weather and atmospheric radiation at ground level. It is an observatory designed, built and operated by the LAGO Collaboration, a non-centralized collaborative union of more than 30 institutions from ten countries.

In this paper we describe the scientific and academic goals of the LAGO project - illustrating its present status with some recent results - and outline its future perspectives.
\end{abstract}

\section{Introduction}

Astroparticle physics is nowadays one of the scientific fields that evidence large interdisciplinary contributions. This is not only possible but even needed, given the large array of topics this discipline covers. Several spaceborne and ground-based cosmic ray observatories have been built or are under development. These facilities are mainly coordinated in large international collaborations with hundreds or even thousands of scientists. In the particular case of Latin America (LA), the successful installation and commissioning of the Pierre Auger Observatory [1] in Malargüe, Argentina, generated an outstanding opportunity to develop Astroparticle physics and High Energy Physics in this region. In this work, we describe one of the resulting projects of this regional development: the Latin American Giant Observatory (LAGO).

\section{The Latin American Giant Observatory}

The Latin American Giant Observatory (LAGO), formerly known as the Large Aperture Gamma Ray Bursts Observatory, is a project conceived in 2006 [2] to detect the high energy component of Gamma Ray Bursts (GRBs, with typical energy of primaries $E_{p} \gtrsim 20 \mathrm{GeV}$ ) by installing $10 \mathrm{~m}^{2}-20 \mathrm{~m}^{2}$ water Cherenkov detectors (WCD) at very high altitude sites across the Andean ranges. From this initial aim, LAGO has evolved toward an extended astroparticle observatory at a regional scale, currently operating WCDs and other particle detectors at nine countries in LA (Argentina, Bolivia, Brazil, Colombia, Ecuador, Guatemala, Mexico, Peru and Venezuela) together with the recent incorporation of institutions from Spain. LAGO is operated by the LAGO Collaboration, a cooperative and non-centralized collaboration of 26 institutions. 
LAGO has three main scientific objectives: to study high energy gamma events at high altitude sites, to understand space weather phenomena through monitoring at the continental scale, and to decipher the impact (direct and indirect) of the cosmic radiation on atmospheric phenomena. These objectives are complemented by two main academic goals: to train students in astroparticle and high energy physics techniques, and foremost, to support the development of astroparticle physics in LA [3].

The LAGO detection network, consist of single or small arrays of astroparticle detectors installed in different sites across the Andean region [4]. Currently, ten WCDs are in operation and we expect to have another eleven detectors starting their operation and calibration in the next biennium. Our detection network spans a region from the south of Mexico, with a small array installed at Sierra Negra (4550 m a.s.1.), to Patagonia, with three WCDs installed at Bariloche (865 $\mathrm{m}$ a.s.1.). In addition, the installation of two WCDs was recently funded for the Marambio Base (Arg., 200m a.s.1.) located in the Antarctic Peninsula, mainly oriented for Space Weather studies and monitoring [5].

The network is distributed over similar geographical longitudes but a wide range of geographical latitudes and altitudes. By combining simultaneous measurements at different rigidity cutoffs from regions with differing atmospheric absorption we are able to produce near-real-time information at different energy ranges of, for example, disturbances induced by interplanetary transients and long term space weather phenomena.

Each LAGO water Cherenkov detector consists of a plastic tank containing $1 \mathrm{~m}^{3}$ to $40 \mathrm{~m}^{3}$ of purified water, where one to four top-mounted large photomultiplier tubes (PMT) collect the Cherenkov light produced by ultra-relativistic particles moving through the water volume. Typically, three types of large photocatode area PMTs are used in LAGO WCDs: Photonis XP1805, Hamamatsu R5912 and Electron Tubes 9353KB. The water purification protocol used includes physical (membranes filtration and sedimentation); chemical (mainly coagulation and flocculation using alum, $\mathrm{Al}_{2} \mathrm{SO}_{4}$, and iron salts, $\mathrm{FeCl}_{3}$ ); and biological methods (activated carbon filtration and disinfectant agents such as $\mathrm{NaClO}$ ). With this method we achieve very low turbidity and high purity water, with a mean resistivity of the order of $\sim \mathrm{M} \Omega$. An internal coating of an ultra-violet light highly reflective and diffusive fabric is used. The WCD signals are shaped and digitized by a custom made $40 \mathrm{MHz}$ electronic board controlled by a Digilent Nexys2 FPGA.

Besides WCD signals, the need to measure very different physical magnitudes, such as cloudiness, humidity and atmospheric pressure and temperature, in remote places with almost no infrastructure requires autonomous and reliable detectors and components. We have moreover based our design in commercial off-the-shelf devices, which have to be available in all the countries where LAGO operates [4]. The main component is the electronic system that operates the complete station. Additional sensors are integrated in an Arduino controlled common sensor module to measure atmospheric pressure, air temperature, solar radiance, cloudiness, and even greenhouse and smog gas concentrations. A commercial global navigation satellite system module is included for timing synchronization and to allow ionospheric total electron content (TEC) calculations at some sites [6]. All these devices, including the WCD electronics boards, are powered by solar panels and are controlled by a single board computer that includes a GPRS module to transfer telemetry and on board pre-analyzed data by using mobile phone networking data services. This new concept produces data that extends the typical objectives of a cosmic ray observatory, allowing several scientific, academic and even citizen science communities to take advantage of our regional scale detection network [3].

\section{The LAGO Programs}

Scientific and academic objectives are organized in different programs and are carried out by the corresponding working groups. LAGO programs cover several aspects of the project, from the installation, calibration and operation of the detectors to the search for pathways to transfer data from remote sites. Complete simulation chains involving all the related aspects (from CR propagation to detector response) [5], data analysis techniques specially designed for the very different energy and temporal scales of the studying phenomena [5, 7], data preservation, and the design of new experiments to be conducted in graduate and undergraduate lectures in the participating Universities, are just some examples of the range of the objectives of the LAGO project.

High altitude sites $(h>4500 \mathrm{~m})$ are designed and operated mainly for the search for high energy components of GRB. Such sites are chosen to diminish the atmospheric absorption of extensive air showers (EAS) initiated by low energy cosmic rays. To increase electromagnetic-muon separation at single pulse level in high-altitude sites, a method based on the total charge and pulse rise-time analysis is also implemented. The increase in separation performance can be used to improve the search for possible GRB candidates, as gamma initiated showers show lower muon fractions at detector level when compared with hadronic primaries.

All the LAGO programs exploit the single particle technique (SPT) by looking for significant excesses in the counting of background signals at different sites. In particular, the LAGO facilities at Chacaltaya mountain consist of three LAGO WCDs with a total detection area of $10 \mathrm{~m}^{2}$. In the period 2010-2012 the Chacaltaya station has collected data during $\sim 17000$ hours of detection for WCD1 and WCD2, and $\sim 15700$ hours for WCD3. Recent studies, based on CORSIKA simulations, show that the angular aperture of the Chacaltaya site 

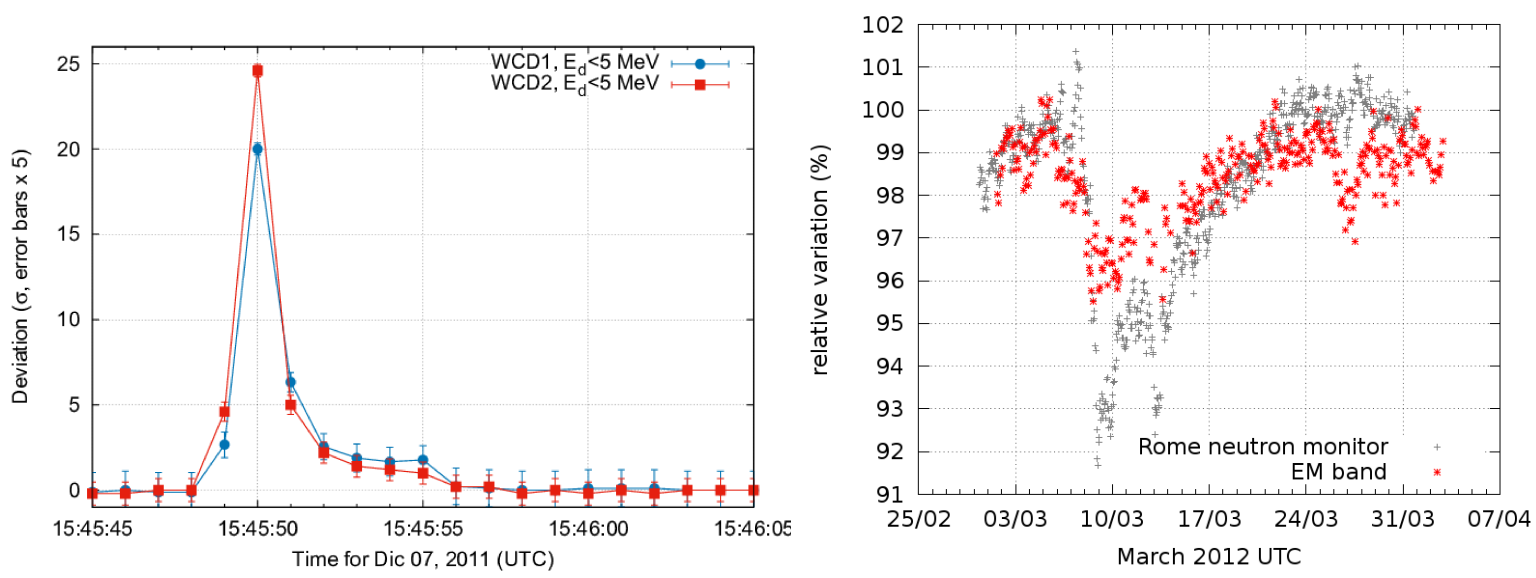

Figure 1: Left: Signal alert for a potential event candidate registered on Wed Dec 07 15:47:02.378 UTC 2011 detected by LAGO at Chacaltaya, Bolivia, $5250 \mathrm{~m}$ a.s.l. (from [7]). Right: Multi-spectral analysis of the Forbush Decrease of March 8th, 2012, measured in a single $1.8 \mathrm{~m}^{2}$ WCD in Bariloche, Argentina (red stars), compared with neutron flux measured by the Rome neutron monitor (gray pluses).

can be extended up to a zenith angle of $25^{\circ}$ in the energy range of interest for GRB and other Gamma originated signals. We estimate that detection area should be ten times larger to extend sensitivity up to $45^{\circ}$. Combining these results with the uptime of each WCD in this period, the total exposure of this site accumulated during this period was $2.7 \times 10^{8} \mathrm{~m}^{2} \mathrm{~s}$ sr. Considering the typical variations of the flux at the time scales of interest, we produced a strict search criterion based on an adaptive moving window averaging method (see [7]). Following this procedure, a new potential candidate was found, appearing on Wed Dec 07 15:45:49.675 \pm 0.005 UTC 2011 and a duration $5.5 \mathrm{~s}$, as can be seen in the left panel of figure 1 . The equatorial coordinates of Chacaltaya zenith were RA/Dec (J2000) $16^{\mathrm{h}} 17^{\mathrm{m}} 31.3^{\mathrm{s}} /-16^{\circ} 21^{\prime} 00^{\prime \prime}$, with an acceptance aperture of $\theta \lesssim 25^{\circ}$. At that time, the Fermi satellite was outside of our acceptance cone in Chacaltaya.

While this technique has the lack of directional reconstruction, it is however possible to use atmospheric absorption as a selection tool for periodic signals including gamma point sources. Data stacking or summation in two different time systems, solar and sidereal, were made over the data. The idea behind this process is based on the random, Poissonian, nature of the majority of the radiation measured by our detectors. When a non random and periodic signal exists, data can be summed over repetitive occurrences, both in sidereal and solar time, to increase the signal-to-noise ratio. After doing this, we observed clear indications, both in phase and in amplitude, of solar modulation of the flux of galactic cosmic rays. These observations demonstrate that, by using an adapted analysis technique to the characteristics of our small detectors, it is possible to observe space weather phenomena at different time scales from ground level in the LAGO network of WCD.

Simultaneous measurements of galactic cosmic ray flux modulation at different locations on Earth using the same type of detector can provide important information on the properties and global structure of magnetic clouds reaching the terrestrial environment during interplanetary Coronal Mass Ejections (ICME) events pointing towards Earth [8]. By using WCDs, we are able to determine the flux of secondary particles at different bands of deposited energy within the detector volume. These bands are dominated by different components of the CR spectrum reaching the Earth's atmosphere (primaries). This multi-spectral analysis technique (MSAT) constitutes the basis of our Space Weather oriented data analysis [5]. By combining all the data measured at different locations of the detection network, the LAGO project will provide very detailed and simultaneous information of the temporal evolution and small- and large-scale characteristics of the disturbances produced by different transient and long-term space weather phenomena.

A complex and complete chain of simulations support this program [5]. For every LAGO site, the directional rigidity cutoff $R_{c}$ is determined for secular and altered geomagnetic field conditions, such as those produced during intense geomagnetic storms. The expected number of primaries is then determined by integrating the measured flux of all the hadronic cosmic rays with $1<E_{p} / \mathrm{GeV}<10^{6}$. A set of CORSIKA simulations is then used to determine the expected number of secondary particles at ground level. Only those secondaries originating from primaries with rigidities above local cut-offs are included. Finally, the expected secondary particles are injected into a Geant4-based model of the LAGO WCD to obtain the signal light flux at the detector level.

With the LAGO MSAT we are able to determine the evolution of the flux observed in three different bands. As the transition points between these different regimes are characterized by changes in the histogram slopes, a fully automated algorithm searches for all these features in 1-hour calibration histograms. As an example, in the right panel of figure 1 the measurement of the Forbush decrease occurred on March 8th, 2012, is shown for 
the electromagnetic dominated band. These measurements demonstrate the capabilities of WCDs to extend present studies of space weather phenomena using low cost detectors from ground level.

\section{Conclusions}

In this paper we outline the Latin American Giant Observatory, its main scientific capabilities and implications for the development of astroparticle physics at Latin America. We also describe the key programs of LAGO, oriented to search for GRBs, high energy phenomena, and space weather, using low cost and reliable detectors at ground level. A complete chain of simulations and data analysis techniques has been developed to exploit WCD measuring capabilities in different energy regimes. Several cited examples demonstrate the LAGO capabilities to study very different astrophysical phenomena. With new detectors coming into operation (for example at as the new LAGO site in Antarctica) a large and unprecedented detection network is emerging at a regional scale.

Besides its scientific objectives, the LAGO project is also a seeder for astroparticle physics development in the LA. A proof of this is the fact that most of the results described in this work have been obtained by several undergraduate and master students working in coordinated collaboration in several Latin American countries. LAGO is a promising observatory that is helping to support the development of several interdisciplinary branches associated with Astroparticles in Latin America.

Acknowledgments The LAGO Collaboration is very thankful to all the participating institutions and to the Pierre Auger Collaboration for their continuous support.

\section{References}

[1] The Pierre Auger Collaboration, The Pierre Auger Cosmic Ray Observatory (2015) 197doi:10.1016/j.nima.2015.06.058.

[2] D. Allard, I. Allekotte, C. Alvarez, et al., Use of water-Cherenkov detectors to detect Gamma Ray Bursts at the Large Aperture GRB Observatory (LAGO), Nuclear Instruments and Methods in Physics Research Section A 595 (1) (2008) 70-72. doi:10.1016/j.nima.2008.07.041.

[3] H. Asorey, for the LAGO Collaboration, LAGO: the Latin American Giant Observatory, in: Proceedings of Science, The Hague, 2015, p. PoS(ICRC2015)247.

[4] I. Sidelnik, for the LAGO Collaboration, The Sites of the Latin American Giant Observatory, in: Proceedings of Science, The Hague, 2015, p. PoS(ICRC2015)665.

[5] H. Asorey, S. Dasso, L. A. Núñez, et al., The LAGO Space Weather Program: Directional Geomagnetic Effects, Background Fluence Calculations and Multi-Spectral Data Analysis, Proceedings of Science ICRC2015 (2015) PoS(ICRC2015)142.

[6] A. J. Mannucci, B. D. Wilson, et al., A global mapping technique for GPS-derived ionospheric total electron content measurements, Radio Science 33 (3) (1998) 565-582. doi:10.1029/97RS02707.

[7] H. Asorey, P. Miranda, A. Núñez-Castiñeyra, L. A. Núñez, J. Salinas, C. Sarmiento Cano, R. Ticona, A. Velarde, Analysis of Background Cosmic Ray Rate in the 2010-2012 Period from the LAGO-Chacaltaya Detectors, in: Proceedings of Science, The Hague, 2015, p. PoS(ICRC2015) 414.

[8] K. Munakata, Probing the heliosphere with the directional anisotropy of galactic cosmic-ray intensity, Proceedings of the IAU 7 (S286) (2012) 185-194. doi:10.1017/S1743921312004826. 